\title{
Aircraft Position Estimation Comparison of Multilateration System Lateration Algorithms with Different Reference Selection Techniques
}

\begin{abstract}
Abdulmalik Shehu Yaro
Department of Communications Engineering, Ahmadu Bello University, Zaria, Nigeria.

*Corresponding author: yaroabdulmalik@yahoo.com, Tel: +2348069254680

Abstract: The lateration algorithm is used by the multilateration (MLAT) system to estimation the position of an aircraft using a time difference of arrival (TDOA) measurement vector generated from its transponder emissions detected at spatially deployed ground station (GS)s. The accuracy at which the aircraft position is determined by the lateration algorithm depends on several factors two of which are the choice and number of reference GSs used in generating the TDOA measurement vector. This paper compares the aircraft PE accuracy of a lateration algorithm using TDOA measurement vector generated using a single and double GS reference selection techniques which are respectively based on signal-to-noise (SNR) and condition number computations. The PE accuracy comparison is carried out at some selected aircraft positions with the GSs in a square configuration distribution. Monte Carlo (MC) simulation result shows that the PE error obtained by the lateration algorithm using TDOA measurement vector generated with the double GS reference selection technique is on the average $20 \%$ less than that obtained using the single GS reference selection technique.
\end{abstract}

Keywords: Reference selection, Condition number, Signal-to-Noise ratio, Lateration algorithm, Position estimation

Article History: received 23 January 2019; accepted 10 April 2019; published 25 April 2019.

(C) 2019 Penerbit UTM Press. All rights reserved

\section{INTRODUCTION}

This document is a template for Microsoft Word. Structure Multilateration (MLAT) system is one of the wireless positioning systems used by the air navigation service provider (ANSP) for surveillance purposes [1]. It consists of spatially located ground stations (GS)s connected to a central processing station (CPS) where the aircraft position estimation (PE) process takes place. The aircraft PE process of the MLAT system is in two stages [2, 3]. The first stage involves generating a time difference of arrival (TDOA) measurement vector using the aircraft transponder emission (at $1090 \mathrm{MHz}$ ) detected at each of the spatially deployed GSs [3-5]. In the second stage which is the scope of this paper, the generated TDOA measurement vector from the first stage is used by the lateration algorithm with the known coordinates of the deployed GSs to estimate the position of the aircraft.

Each of the TDOAs in the measurement vector generated in the first stage have a non-linear relationship with the position of the aircraft [6-8]. Thus, several approaches to establish a linear relationship between the two variables have been presented which resulted in the two forms of lateration algorithms namely closed-form and open-form [8]. The open-form lateration algorithm is mostly used when the MLAT system is in an active aircraft PE mode. In this scenario, the MLAT system is not standalone. It is installed alone with other surveillance systems such as surveillance radar and automatic dependent surveillance broadcast (ADS-B). The closedform lateration algorithm is used when the MLAT system is in a passive mode (standalone) which is considered in this paper for aircraft PE.

The linearization process in the closed-form approach involves algebraic manipulation of the TDOA equation also known as hyperbolic equation to establish a linear relationship between the two variables resulting in what is known as plane equations [8, 9]. The algebraic manipulation made the closed-form lateration algorithm have high PE error due to its high sensitivity to errors in the input variable [7]. Several works have proposed different techniques to improve the PE accuracy of the closed-form lateration algorithm through the reduction of the PE error $[6,8,10-12]$. One of the techniques considered is choosing the suitable GS reference to generate the TDOA measurement vector for use by the lateration algorithm $[8,10,13]$. In [9], a condition numberbased GS reference pair selection technique is proposed. It involves solving and choosing the GS pair whose TDOA measurement vector resulted in the least condition number value of a TDOA measurement-based matrix. Another technique to selecting a suitable GS reference is the signalto-noise ratio (SNR) based technique [11]. It involves choosing a GS that is closest to the emitting aircraft as the reference in generating the TDOA measurement vector. Both techniques have been shown to improve the PE accuracy of the closed-form lateration algorithm. 
However, no aircraft PE accuracy comparison between the two techniques have been presented to determine the best. Thus, in this paper, the aircraft PE accuracy comparison of the lateration algorithm using the TDOA measurement vector generated with the single GS reference selection technique based on SNR computation and double GS reference selection technique based on condition number computation is presented. This is to determine which of the GS reference selection technique who's generated TDOA measurement vector resulted in the least aircraft PE error by the closed-form lateration algorithm.

The reminder of the paper is organised as follows. Section 2 presents a brief description of the closed-form lateration algorithms based on single and double referencing GS approaches. The GS reference selection techniques to be used with the lateration algorithms is presented in Section 3 while the simulation result and discussion are presented in Section 4. Finally, the conclusion is presented in Section 5. the paper into sections and subsections, including the opening brief abstract, introduction, body and summary. Include a list of references at the end of the chapter.

\section{AIRCRAFT POSITION ESTIMATION METHODOLOGY}

In this section of the paper, the mathematical derivations of the lateration algorithms based on the single and double GS referencing approaches are presented.

\subsection{Single Ground Station Reference Close-form Lateration Algorithm}

Let $\mathbf{x}_{e}=[x, y]^{T}$ be the position of an aircraft while $\mathbf{s}_{i}=$ $\left[x_{i}, y_{i}\right], \mathbf{s}_{j}=\left[x_{j}, y_{j}\right], \mathbf{s}_{k}=\left[x_{k}, y_{k}\right]$ and $\mathbf{s}_{m}=\left[x_{m}, y_{m}\right]$ respectively be the locations of the $i^{\text {th }}, j^{\text {th }}, k^{\text {th }}$ and $m^{\text {th }}$ GSs. Using the $i^{\text {th }} \mathrm{GS}$ as the reference station to generate the TDOA measurement vector, three hyperbolic equations that relates the TDOAs with the aircraft position are obtained and presented as follows [10]:

$$
\begin{aligned}
& \tau_{i j}=\frac{1}{c}\left(\sqrt{\left(x-x_{i}\right)^{2}+\left(y-y_{i}\right)^{2}}-\sqrt{\left(x-x_{j}\right)^{2}+\left(y-y_{j}\right)^{2}}\right) \\
& \tau_{i k}=\frac{1}{c}\left(\sqrt{\left(x-x_{i}\right)^{2}+\left(y-y_{i}\right)^{2}}-\sqrt{\left(x-x_{k}\right)^{2}+\left(y-y_{k}\right)^{2}}\right) \\
& \tau_{i m}=\frac{1}{c}\left(\sqrt{\left(x-x_{i}\right)^{2}+\left(y-y_{i}\right)^{2}}-\sqrt{\left(x-x_{m}\right)^{2}+\left(y-y_{m}\right)^{2}}\right)
\end{aligned}
$$

where $c=3 \times 10^{5} \mathrm{~km} / \mathrm{s}$

Algebraic manipulation of Eq. (1) to Eq. (3) as performed in [10] results in two plane equations as shown in Eq. (4) and Eq. (5).

$$
\begin{aligned}
& A_{i j k} x+B_{i j k} y=C_{i j k} \\
& A_{i j m} x+B_{i j m} y=C_{i j m}
\end{aligned}
$$

where the coefficients of Eq. (4) and Eq. (5) can be found in [10].

The subscript "ijk" and "ijm" in the coefficients of Eq. (4) and Eq. (5) respectively indicates that variable is obtained using the $i^{\text {th }}$ GS as reference with $j^{\text {th }}-k^{\text {th }}$ pair and $j^{\text {th }}-m^{\text {th }}$ pair as non-reference GS pairs. The Eq. (5) and Eq. (6) can be represented in matrix form as shown in Eq. (6).

$$
\left[\begin{array}{ll}
A_{i j k} & B_{i j k} \\
A_{i j m} & B_{i j m}
\end{array}\right] \cdot\left[\begin{array}{l}
x \\
y
\end{array}\right]=\left[\begin{array}{l}
C_{i j k} \\
C_{i j m}
\end{array}\right]
$$

$$
\mathbf{Q}_{\text {single }} \mathbf{x}_{e}=\mathbf{n}_{\text {single }}
$$

Eq. (6) is the mathematical expression for the aircraft $P E$ process of the closed-form lateration algorithm based on single GS referencing.

\subsection{Double Ground Station Reference Closed-form Lateration Algorithm}

Unlike in the single GS reference lateration algorithm in which a single GS is used a reference, a pair of GS is used in generating the TDOA measurement vector in the double GS reference closed-form lateration algorithm. Choosing the ith and jth GSs a reference pair with the kth and mth as non-reference GSs, four TDOA hyperbolic equations are generated as follows [9].

$$
\begin{aligned}
& \tau_{i k}=\frac{1}{c}\left(\sqrt{\left(x-x_{i}\right)^{2}+\left(y-y_{i}\right)^{2}}-\sqrt{\left(x-x_{k}\right)^{2}+\left(y-y_{k}\right)^{2}}\right) \\
& \tau_{j k}=\frac{1}{c}\left(\sqrt{\left(x-x_{j}\right)^{2}+\left(y-y_{j}\right)^{2}}-\sqrt{\left(x-x_{k}\right)^{2}+\left(y-y_{k}\right)^{2}}\right) \\
& \tau_{i m}=\frac{1}{c}\left(\sqrt{\left(x-x_{i}\right)^{2}+\left(y-y_{i}\right)^{2}}-\sqrt{\left(x-x_{m}\right)^{2}+\left(y-y_{m}\right)^{2}}\right) \\
& \tau_{j m}=\frac{1}{c}\left(\sqrt{\left(x-x_{j}\right)^{2}+\left(y-y_{j}\right)^{2}}-\sqrt{\left(x-x_{m}\right)^{2}+\left(y-y_{m}\right)^{2}}\right)
\end{aligned}
$$

Algebraically manipulating Eq. (7) to Eq. (10) results in two plane equations as follows:

$$
\begin{aligned}
& \bar{A}_{i j k} x+\bar{B}_{i j k} y=\bar{C}_{i j k} \\
& \bar{A}_{i j m} x+\bar{B}_{i j m} y=\bar{C}_{i j m}
\end{aligned}
$$

where the coefficients of Eq. (11) and Eq. (12) are functions of TDOA measurements and GS coordinates which can be found in [9].

Subsequently, the representation of Eq. (11) and Eq. (12) in matrix form is as follows:

$$
\begin{aligned}
& {\left[\begin{array}{cc}
\bar{A}_{i j k} & \bar{B}_{i j k} \\
\bar{A}_{i j m} & \bar{B}_{i j m}
\end{array}\right] \cdot\left[\begin{array}{l}
x \\
y
\end{array}\right]=\left[\begin{array}{l}
\bar{C}_{i j k} \\
\bar{C}_{i j m}
\end{array}\right]} \\
& \mathbf{Q}_{\text {double }} \mathbf{x}_{e}=\mathbf{n}_{\text {double }}
\end{aligned}
$$


Eq. (13) is the mathematical expression for the aircraft PE process of the closed-form lateration algorithm based on double GS reference.

\subsection{Total Least Square (TLS) Singular Value Decomposition (SVD)}

In this subsection, the total least square (TLS) approach based on singular value decomposition (SVD) used in finding the solution to the least square (LS) problems in Eq. (6) and Eq. (13) is presented. Let matrix $\mathbf{M}$ be a concatenation of matrix $\mathbf{Q}$ and $\mathbf{n}$ in either Eq. (6) or Eq. (13) as follows:

$\mathbf{M}=\left[\begin{array}{ll}\mathbf{Q}_{y} & \mathbf{n}_{y}\end{array}\right]$

where $y \in[$ single, double $]$

Taking the SVD of matrix $\mathbf{M}$ as follows [15]:

$\mathbf{M}=\mathbf{U} \mathbf{\Sigma} \mathbf{V}^{\mathbf{T}}=\sum_{i=1}^{3} u_{i} \sigma_{i} v_{i}^{T}$

The estimated position of the aircraft by the closed-form lateration algorithm is obtained as:

$\hat{\mathbf{x}}_{e}=\left[\begin{array}{c}\hat{x} \\ \hat{y}\end{array}\right]=\frac{-1}{v_{3,3}}\left[v_{1,3}, v_{2,3}\right]^{T}$

Eq. (16) is the estimate location of the aircraft using either Eq. (6) or Eq. (13). It is assumed the TDOA error is the major cause of the PE error which is modelled as a Gaussian random variable with probability density function as $N\left(0, \tau_{\sigma}\right)$ where $\tau_{\sigma}$ is known as the TDOA error standard deviation (SD) given in seconds.

\section{GROUND STATION REFERENCE SELECTION TECHNIQUES}

In this section of the paper, the GS reference selection techniques to be used with the single and double GS reference closed-form lateration algorithms presented in Section 2.1 and Section 2.2 for the aircraft PE are presented.

\subsection{Single Ground Station Reference Selection Technique}

For the single GS reference selection technique, the SNRbased method proposed in [11] is used. Let $P_{t}$ in $\mathrm{dBm}$ be the aircraft transponder transmit power of the signal which is detected at each of the deployed GSs. The received aircraft transponder emission power in $\mathrm{dBm}$ at any of the GS is obtained as follows:

$P_{r}^{w}=P_{t}+G_{t}+G_{r}-P L^{w}$

where $w \in[i, j, k, m]$ and $P L^{w}$ is the path loss attenuation on the aircraft transponder emission obtained using Eq. (18).

$P L^{w}=32.44+20 \log _{10}\left(R_{w}\right)+20 \log _{10}\left(f_{M H z}\right)$

The $R_{w}$ in Eq. (18) is the horizontal distance between the aircraft and any of the GS. Let $P_{n}$ be the receiver sensitivity used at each of the GS, then the SNR of the received aircraft transponder emission at any of the GS is:

$S N R^{w}=P_{r}^{w}-P_{n}$

Using Eq. (19), the GS to be used as reference in generating the TDOA measurement vector is determined and it is the GS with the least SNR value. That is, the GS with least distance from the aircraft.

\subsection{Double Ground Station Reference Selection Technique}

The GS reference selection technique proposed in [9] is used in determining the suitable GS pair as reference for the lateration algorithm in Section 2.2. It involves calculating the condition number of a TDOA measurement-based matrix and using the GS pair with the least condition number as reference to generate the TDOA measurement vector. The TDOA measurement matrix is expressed as follows [9]:

$\mathbf{N}_{i j}=\left[\begin{array}{cc}\tau_{i m} \times \tau_{i k} & 0 \\ 0 & \tau_{j m} \times \tau_{j k}\end{array}\right]$

The condition number of matrix $\mathbf{N}_{i j}$ in Eq. (20) is obtained as follows:

$$
K\left(\mathbf{N}_{i j}\right)=\left\|\mathbf{N}_{i j}\right\|_{2} \cdot\left\|\mathbf{N}_{i j}^{-1}\right\|_{2}
$$

where $\left\|\mathbf{N}_{i j}\right\|$ and $\left\|\mathbf{N}_{i j}^{-1}\right\|$ is the spectra-norm of matrix and its inverse respectively.

Summary of the procedure to select the suitable GS pair to generate the TDOA measurement vector for use with the closed-form lateration algorithm in Section 2.2 is presented below [9]:

Step-1: Generate the TDOA measurement vector for each of the possible GS pair combinations.

Step-2: Solve for $K\left(\mathbf{N}_{i j}\right)$ in Eq. (21) using each of the TDOA measurement vector generated in Step-1.

Step-3: The GS pair whose TDOA measurement vector resulted in the least value of $K\left(\mathbf{N}_{i j}\right)$ in Eq. (21) is the suitable GS reference.

In the next section, the PE accuracy of the two lateration algorithms presented in Section 2 using the GS reference selection techniques in Section 3 is presented.

\section{SIMULATION RESULT AND DISCUSSION}

Position root mean square error (RMSE) is used as the performance measure in determining the PE accuracy of the lateration algorithms. Mathematically, for the $N=500$ realization Monte Carlo (MC) simulation, the position RMSE is mathematically expressed as:

$$
P E_{\text {rmse }}=\sqrt{\frac{1}{N} \sum_{n=1}^{N}\left[\left(x-\hat{x}_{n}\right)^{2}+\left(y-\hat{y}_{n}\right)^{2}\right]}
$$


where $(x, y)$ and $\left(\hat{x}_{n}, \hat{y}_{n}\right)$ respectively are the known and estimate position of the emitter at the $n^{\text {th }}$ MC realization.

The transmitter (aircraft transponder) and receiver parameters for the computation of the SNR value at each of the GS is presented in Table 1 and are based on actual system used in the civil aviation industry.

Table 1. Aircraft transponder and receiver parameters $[15,16]$

\begin{tabular}{|c|c|c|}
\hline \multirow{2}{|c|}{ Parameter } & Values \\
\hline \multirow{2}{*}{$\begin{array}{c}\text { Aircraft } \\
\text { transponder }\end{array}$} & Antenna gain & $3 \mathrm{dBi}$ \\
\cline { 2 - 3 } & Transmit power & $250 \mathrm{~W}$ \\
\cline { 2 - 3 } & Downlink frequency & $1090 \mathrm{MHz}$ \\
\hline \multirow{2}{*}{ GS receiver } & Antenna gain & $12 \mathrm{dBi}$ \\
\cline { 2 - 3 } & Sensitivity & $-90 \mathrm{dBm}$ \\
\hline
\end{tabular}

The GSs used are labelled GS-1, GS-2, GS-3 and GS-4, and are deployed in a square configuration as shown in Figure 1.

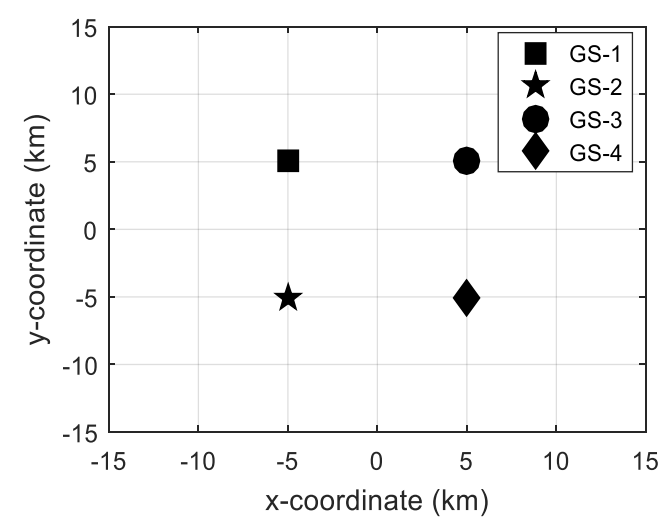

Figure 1. Distribution of the GSs used for the analysis

The PE performance analysis is carried out at six randomly selected aircraft positions with coordinates presented in Table 2 .

Table 2. Selected aircraft positions for analysis

\begin{tabular}{|c|c|c|c|c|c|c|}
\hline \multirow{2}{*}{ Coordinates } & \multicolumn{6}{|c|}{ Aircraft position } \\
\cline { 2 - 7 } & A & B & C & D & E & F \\
\hline$x(\mathrm{~km})$ & 10 & 20 & 40 & 50 & 60 & 90 \\
\hline$y(\mathrm{~km})$ & 30 & 20 & 15 & 80 & 60 & 40 \\
\hline
\end{tabular}

By varying the TDOA error SD from 0 to $2 \mathrm{nsec}$, the position RMSE based on Eq. (22) for the single and double GS reference lateration algorithms each with its corresponding GS reference selection technique is determined at each of the selected aircraft positions in Table 2 and are presented in Figure 2. From Figure 2, it can be seen that irrespective of the lateration algorithm and GS reference selection technique, the position RMSE varies with the location of the aircraft. The lateration algorithm with the double GS reference has the least position RMSE compared to single GS reference. Table 3 shows the position RMSE comparison between the single and double GS reference lateration algorithm at TDOA error SD of $1 \mathrm{nsec}$. At the aircraft position A, the single GS reference lateration algorithm has a position RMSE of about $16.31 \mathrm{~m}$ while that of the double GS reference has about 11.92. The absolute position RMSE difference is about $4.39 \mathrm{~m}$ which corresponds to a reduction of about $27 \%$. This means that at aircraft position A, the double GS reference lateration algorithm produced position RMSE, which is $27 \%$ less than that obtained using the single GS reference lateration algorithm.

The analysis is extended to aircraft at positions B, C, D, $\mathrm{E}$ and $\mathrm{F}$. The absolute position RMSE differences between the single and double GS reference lateration algorithms at aircraft positions $\mathrm{B}, \mathrm{C}, \mathrm{D}, \mathrm{E}$ and $\mathrm{F}$ are $2.39 \mathrm{~m}, 9.18 \mathrm{~m}$, $42.21 \mathrm{~m}, 31.69 \mathrm{~m}$ and $47.50 \mathrm{~m}$ respectively. This corresponds to a percentage absolute difference of about $30 \%, 31 \%, 41 \%, 42 \%$ and $36 \%$ respectively. On the average, based on the selected aircraft positions with coordinates as presented in Table 2 and GS configuration and distribution as presented in Figure 1, the double GS reference lateration algorithm in Section 2.2 with the GS reference selection technique in Section 3.2 produced position RMSEs that are about $20 \%$ less than the single GS reference lateration algorithm with the SNR based GS reference selection technique.

Table 3. Position RMSE comparison at TDOA error SD of $1 \mathrm{nsec}$

\begin{tabular}{|c|c|c|c|}
\hline \multirow{2}{*}{$\begin{array}{l}\text { Aircraft } \\
\text { Position }\end{array}$} & \multicolumn{2}{|c|}{$\begin{array}{l}\text { Position RMSE } \\
(\mathrm{m})\end{array}$} & \multirow{2}{*}{$\begin{array}{c}\text { Absolute } \\
\text { position } \\
\text { RMSE } \\
\text { difference } \\
(\mathrm{m}) \\
\end{array}$} \\
\hline & $\begin{array}{l}\text { Single GS } \\
\text { reference }\end{array}$ & $\begin{array}{l}\text { Double GS } \\
\text { reference }\end{array}$ & \\
\hline A & 16.31 & 11.92 & 4.39 \\
\hline B & 7.96 & 5.57 & 2.39 \\
\hline $\mathrm{C}$ & 27.58 & 18.40 & 9.18 \\
\hline $\mathrm{D}$ & 101.50 & 59.29 & 42.21 \\
\hline $\mathrm{E}$ & 74.44 & 42.75 & 31.69 \\
\hline $\mathrm{F}$ & 131.10 & 83.60 & 47.50 \\
\hline
\end{tabular}

\section{CONCLUSION}

In this paper, the PE accuracy of a single and double GS reference lateration algorithms each with a GS reference selection technique is presented. This is aimed at determined the best reference selection technique whose TDOA measurement vector resulted in the least aircraft PE error. The analysis is carried out at some randomly selected aircraft positions with the GSs in a square configuration distribution based on MC simulation. Result comparison shows that the double GS reference lateration algorithm produces the least position RMSE which is on the average $20 \%$ less than that produced by the single GS reference lateration algorithm. It is assumed that the main source of the PE error by the lateration algorithm is the TDOA error which is modelled as a Gaussian random variable with a normal probability density function. 


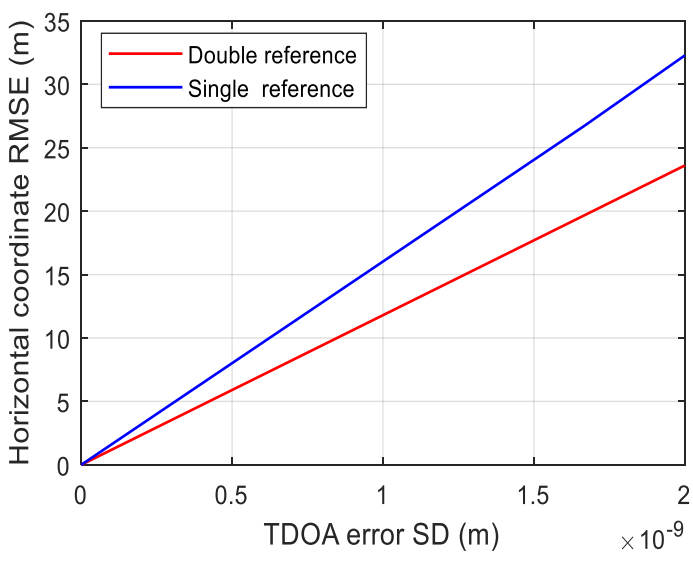

(a) Aircraft Position A

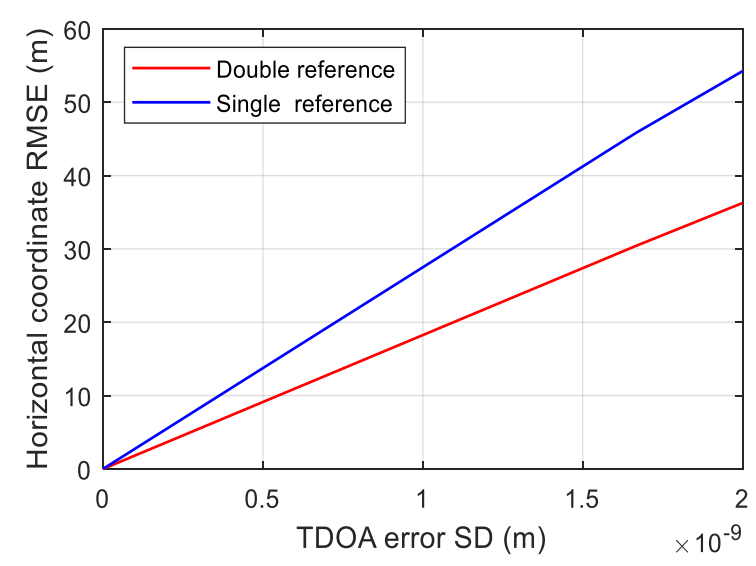

(c) Aircraft Position C

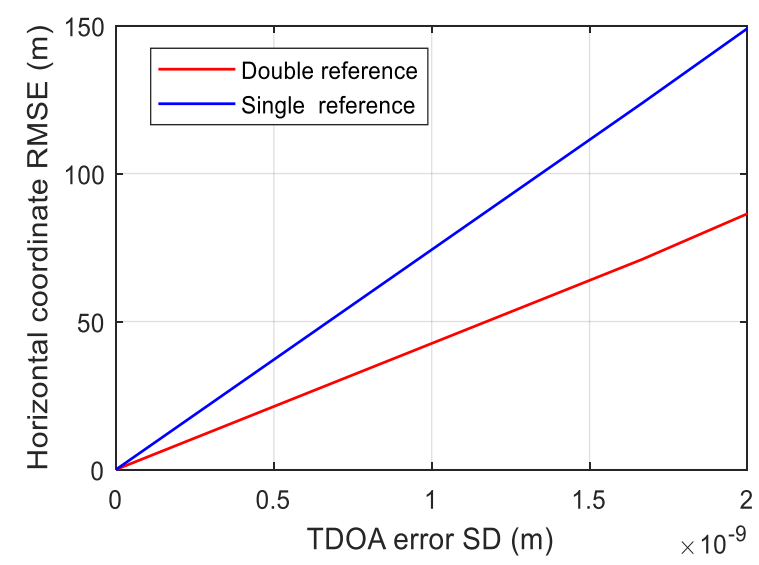

(e) Aircraft Position E

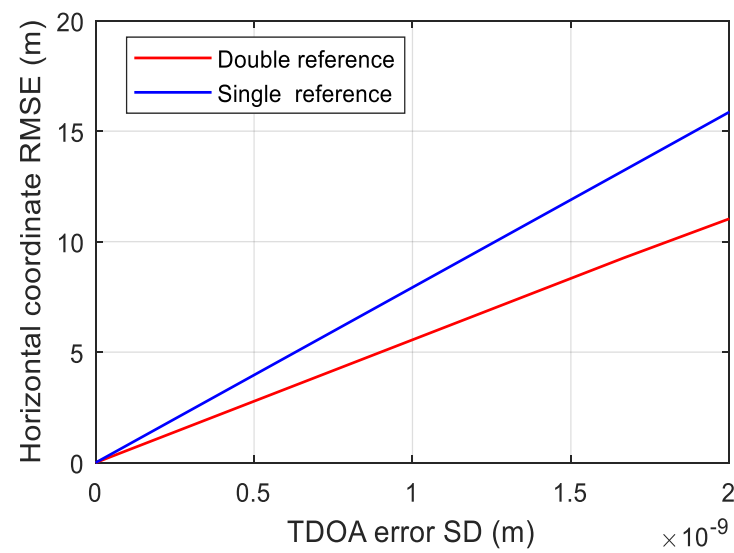

(b) Aircraft Position B

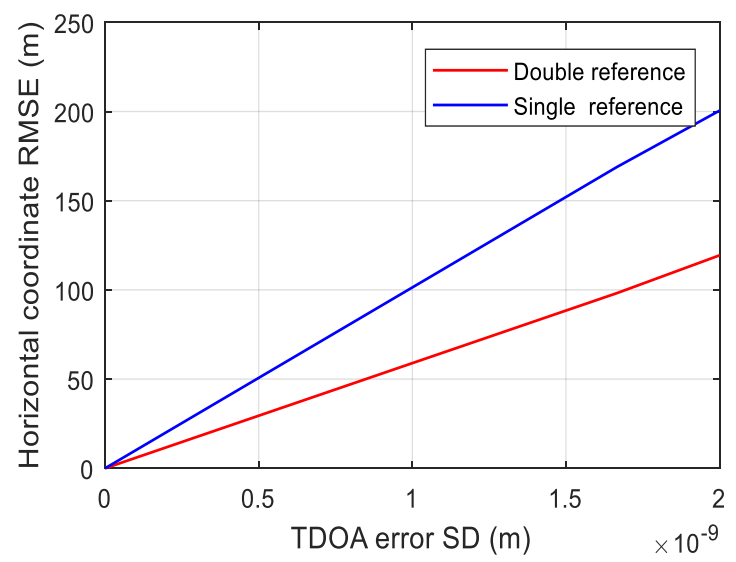

(d) Aircraft Position D

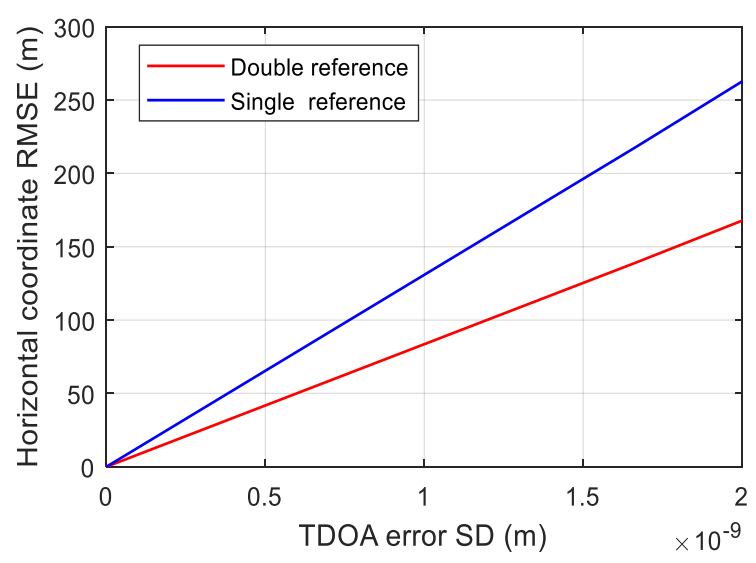

(f) Aircraft Position F

Figure 2. Horizontal coordinate RMSE comparison between the single and double reference lateration algorithm

\section{REFERENCES}

[1] H. L. W. Neven, J. T. Quilter, R. Weedo, and R. A. Hogendoorn, "Wide area multilateration (WAM)," Eurocontrol, Report on EATMP TRS 131/04 Version 1.1, 2005.

[2] B. Huang, L. Xie, and Z. Yang, "TDOA-based source localization with distance-dependent noises," IEEE Trans. Wirel. Commun., vol. 14, no. 1, pp. 468-480, 2015.

[3] A. S. Yaro and A. Z. Sha'ameri, "Emitter velocity estimation comparison for frequency difference of arrival measurement based single and multiple reference lateration algorithm," Elektr. J. Electr. Eng., vol. 17, no. 1, pp. 1-9, 2018.

[4] P. Marmaroli, X. Falourd, and H. Lissek, "A comparative study of time delay estimation techniques for road vehicle tracking," in 11th French Congress of Acoustics and 2012 Annual IOA Meeting, 2012, pp. 136-140.

[5] L. Meng, X. H. Li, W. G. Zhang, and D. Z. Liu, "The generalized cross-correlation Method for time delay estimation of infrasound signal," in Proceedings - 5th International Conference on Instrumentation and 
Measurement, Computer, Communication, and Control, IMCCC 2015, 2016, pp. 1320-1323.

[6] R. Kaune, C. Steffes, S. Rau, W. Konle, and J. Pagel, "Wide area multilateration using ADS-B transponder signals," in 15th IEEE International Conference on Information Fusion, 2012, pp. 727-734.

[7] K. C. Ho, "Bias reduction for an explicit solution of source localization using TDOA," IEEE Trans. Signal Process., vol. 60, no. 5, pp. 2101-2114, 2012.

[8] I. A. Mantilla-Gaviria, M. Leonardi, G. Galati, and J. V. Balbastre-Tejedor, "Localization algorithms for multilateration (MLAT) systems in airport surface surveillance," Signal, Image Video Process., vol. 9, no. 7, pp. 1549-1558, 2015.

[9] A. S. Yaro, A. Z. Sha'ameri, and N. Kamel, "Ground receiving station reference pair selection technique for a minimum configuration $3 \mathrm{D}$ emitter position estimation multilateration system," Adv. Electr. Electron. Eng., vol. 15, no. 3, pp. 391-399, Oct. 2017.

[10] M. Stojilovi, B. Menssen, I. Flintoft, H. Garbe, J. Dawson, and M. Rubinstein, "TDoA-based localisation of radiated IEMI sources," IEEE Int. Symp. Electromagn. Compat., pp. 1263-1268, 2014.

[11] J. E. Rene, D. Ortiz, P. Venegas, and J. Vidal, "Selection of the reference anchor node by using
SNR in TDOA-based positioning," in IEEE Ecuador Technical Chapters Meeting (ETCM), 2016, pp. 1-4.

[12] Y. Weng, W. Xiao, and L. Xie, "Total least squares method for robust source localization in sensor networks using TDOA measurements," Int. J. Distrib. Sens. Networks, vol. 7, no. 1, p. 172902, 2011.

[13] M. D. Gillette and H. F. Silverman, "A linear closedform algorithm for source localization from timedifferences of arrival," IEEE Signal Process. Lett. vol. 15, no. 1, pp. 1-4, 2008.

[14] Q. Xu, Y. Lei, J. Cao, and H. Wei, "An improved algorithm based on reference selection for time difference of arrival location," in 7th International Congress on Image and Signal Processing, CISP 2014, 2014, no. 3, pp. 953-957.

[15] I. Markovsky, D. M. Sima, and S. Van Huffel, "Total least squares methods," Wiley Interdiscip. Rev. Comput. Stat., vol. 2, no. 2, pp. 212-217, Mar. 2010.

[16] R. Francis et al., "The flying laboratory for the observation of ADS-B signals," Int. J. Navig. Obs., vol. 2011, no. 2011, pp. 1-5, 2011.

[17] INDRA, "Wide area multilateration system," 2017. [Online]. Available: https://www.indracompany .com/.../indra-indra_wide_area_multilateration_ system.pdf. [Accessed: 01-Apr-2018]. 